\title{
Espace, temps et théorie du capital chez Henri Lefebvre et
}

Marx

Space, time, and the theory of capital in Henri Lefebvre and

Marx

\section{Espacio, tiempo y teoría del capital según Henri Lefebvre y Marx}

\section{Louis Gaudreau}

Numéro 55, automne 2013

Financiarisation du capitalisme

URI : https://id.erudit.org/iderudit/1027685ar

DOI : https://doi.org/10.7202/1027685ar

Aller au sommaire du numéro

\section{Éditeur(s)}

Athéna éditions

ISSN

0831-1048 (imprimé)

1923-5771 (numérique)

Découvrir la revue

Citer cet article

Gaudreau, L. (2013). Espace, temps et théorie du capital chez Henri Lefebvre et Marx. Cahiers de recherche sociologique, (55), 155-176.

https://doi.org/10.7202/1027685ar
Résumé de l'article

Ce texte propose une réflexion sur la production capitaliste de l'espace. Il présente la théorie d'Henri Lefebvre sur l'espace et tente de mettre en lumière la contribution originale de cet auteur à la compréhension du rapport entre temps et espace dans la reproduction du capitalisme. L'analyse porte plus particulièrement sur le concept d'espace abstrait développé par Lefebvre, dont on retrouve une formulation implicite dans les travaux de Marx, et qui révèle le rôle actif joué par la spatialité dans le procès de valorisation capitaliste. Le concept lefebvrien d'espace abstrait jette ainsi les bases d'une remise en question du primat accordé au temps dans la tradition d'analyse critique du capitalisme. Le texte se conclut par de brèves réflexions sur l'actualité d'une telle conception de l'espace pour saisir la récente financiarisation du capital. 


\title{
Espace, temps et théorie du capital chez Henri Lefebvre et Marx
}

Louis GaUdReAu

\begin{abstract}
S'il ne fait nul doute, dans un monde en pleine urbanisation, en proie aux $\checkmark$ crises monétaires, immobilières, alimentaires et aux conflits territoriaux de toutes sortes, que les questions relatives à l'occupation, à l'usage et au contrôle de l'espace sont d'un intérêt central pour la compréhension des phénomènes sociaux contemporains, le statut que l'on doit accorder à la spatialité dans la théorie sociale est, quant lui, loin de faire consensus. Les principales divergences au sujet de la portée explicative de l'espace concernent, plus fondamentalement, la manière de concevoir son rapport au temps. Au risque de simplifier quelque peu ce débat, deux grandes tendances semblent s'y opposer. D'un côté, on retrouve les théories qui s'inscrivent dans une tradition philosophique puisant ses racines chez Kant et Hegel. Celles-ci posent la supériorité en soi, et par essence, du temps sur l'espace. La temporalité y est ainsi conçue comme le domaine du mouvement, le principe actif de l'histoire et l'unique source du possible. La spatialité y est à l'inverse présentée comme la dimension statique de l'existence qui agit à la manière d'un miroir changeant en immédiateté et en permanence le dynamisme temporel des interactions sociales ${ }^{1}$. L'espace aurait au mieux une fonction descriptive permettant de rendre compte des effets produits par des transformations historiques (temporelles) antérieures. C'est, par exemple, en s'appuyant sur
\end{abstract}


un tel postulat que Georg Lukàcs a formulé sa critique du capitalisme dans Histoire et conscience de classe. Selon lui, le pouvoir de domination du capital résiderait dans sa capacité à inverser le rapport fondamental du temps à l'espace ou plutôt, à vider la temporalité de sa nature qualitative pour lui donner la forme réifiée d'un espace 2 .

Le point de vue opposé a surtout été développé dans le domaine de la géographie où l'on a fait valoir la centralité de l'espace dans la production de la différence et la pluralité nécessaires à toute forme de changement. Dans son livre For Space, Doreen Massey présente une critique systématique de la philosophie qui a eu tendance à associer l'espace à l'inertie et au statisme. Elle plaide en faveur d'une conception alternative de la spatialité qui la reconnaîtrait en tant que sphère où la multiplicité devient possible ${ }^{3}$. Elle y remet également en question le primat naturel habituellement accordé au temps face à l'espace. Elle soutient plutôt que la multiplicité constitutive de l'espace doit être considérée comme l'exigence d'une temporalité dynamique et que c'est uniquement dans le cadre hétérogène résultant de l'interaction entre le temps et l'espace qu'apparaîtrait la possibilité d'un futur transformé ${ }^{4}$. Ainsi, il n'y a pas chez elle, tout comme dans la «géographie postmoderne » d'Edward Soja, de rapport hiérarchique entre ces deux dimensions de l'existence, mais une relation d'interdépendance dialectique ${ }^{5}$.

Le sociologue et philosophe Henri Lefebvre est souvent cité comme l'une des principales sources d'inspiration de cette remise en cause du statisme et de la secondarité de l'espace. Il est en effet bien connu pour sa critique sévère de l'urbanisme scientifique moderne auquel il reprochait de dépouiller l'espace de sa vitalité pour lui substituer une spatialité homogène assujettie aux exigences de l'accumulation capitaliste ${ }^{6}$. En empruntant à Lefebvre l'idée d'une «différencialité» propre aux espaces du quotidien et qui ne peut être saisie par aucun discours universel et unifiant, la géographie

2. Georg Lukàcs Histoire et conscience de classe. Essai de dialectique marxiste, Paris, Les Éditions de Minuit, 1960, $417 \mathrm{p}$.

3. Doreen Massey, For Space, Londres, Sage publications, 2005, p. 11.

4. Ibid., p. 89.

5. Edward W. Soja, Postmodern Geographies. The Reassertion of Space in Critical Social Theory, Londres/New York Verso, 1989, $266 \mathrm{p}$.

6. Lefebvre a développé cette critique dans plusieurs ouvrages consacrés à l'espace et la ville : La révolution urbaine, Le droit à la ville, La production de l'espace, La pensée marxiste et la ville et Du rural à l'urbain. Les trois volumes de la Critique de la vie quotidienne ainsi que le quatrième tome de l'État abordent également cette question. Henri Lefebvre, La révolution urbaine. Paris, Gallimard, coll. «Idées», 1970, 248 p.; Le droit à la ville suivi de Espace et politique, Paris, Anthropos, coll. «Points», 1974, 281 p.; La pensée marxiste et la ville, $3^{e}$ édition, Casterman, coll. «Synthèses contemporaines», 1978, 169 p.; De l'État 4. Les contradictions de l'État moderne. La dialectique et de l'État, Paris, Union générale d'édition, coll. «10/18», 1978, 467 p.; La production de l'espace, Paris, Anthropos, coll. «Ethno-sociologie», 1974/2000, 4 e édition, 485 p.; Du rural à l'urbain. Paris, Anthropos, coll. «Ethnosociologie», 2001, 3e édition, 299 p.; Critique de la vie quotidienne. Tomes 1, 2 et 3, Paris, L'Arche, coll. «Le sens de la marche», 1958-1981. 
postmoderne a cependant eu tendance à délaisser un autre aspect important, et pourtant indissociable, de la pensée spatiale de cet auteur.

Pour Lefebvre, l'espace qui est source de diversité est aussi, simultanément, inertie et homogénéité. Il n'est pas l'un ou l'autre, soit mouvement soit fixation. Il est les deux à la fois et, si la diversité et le dynamisme sont effectivement des traits essentiels de l'espace, ceux-ci ne peuvent exister que dans un rapport essentiel à l'uniformité et l'immobilité. Lefebvre ajoute que ce caractère contradictoire de l'espace n'en est pas une propriété générale, mais qu'il constitue plutôt la marque distinctive d'une spatialité historiquement spécifique produite par le capital. En fait, Lefebvre a retenu de Marx la thèse selon laquelle le développement du capitalisme serait mû par un procès d'abstraction qui absorbe l'activité productive concrète pour en faire du travail abstrait. Il propose de concevoir l'espace - au même titre que le temps d'ailleurs - comme le support essentiel de ce procès et comme le fruit d'une dynamique qui s'approprie la dimension historique de la vie humaine en vue de la convertir en espace abstrait, c'est-à-dire en "l'espace-marchandise » homogène de l'accumulation capitaliste.

Le procès spatial d'abstraction spécifique au capital pose ainsi un rapport hiérarchique entre deux types inséparables de spatialité, l'une sociale-concrète et l'autre abstraite, qui s'exprime dans la domination de la seconde sur la première. Cette dynamique n'entraîne pas l'abolition complète du concret par l'abstrait. Il ne suffit donc pas de démontrer l'existence d'un espace différentiel pour que cessent d'opérer les mécanismes qui l'assujettissent à la spatialité abstraite. De ce constat découle aussi la nécessité de remettre en question le postulat philosophique de la primauté du temps sur l'espace.

Le texte qui suit débutera par un bref exposé des thèses de Lefebvre sur l'espace. Il effectuera par la suite un retour aux œuvres de Marx qui ont grandement marqué la pensée de Lefebvre (Le Capital et les Grundrisse) et dans lesquelles on peut déjà voir à l'œuvre certains éléments de la dialectique spatiale formulée de manière plus explicite par ce dernier. Cette analyse s'intéressera surtout à la description que Marx donne des conditions historiques de l'avènement du capitalisme et de son évolution subséquente, plus spécialement du passage de la soumission formelle à la soumission réelle au capital. Je tenterai d'établir que ces deux stades du développement du capitalisme, dont Marx a principalement fait valoir la dimension temporelle, ont également, et indissociablement, trouvé leurs conditions de possibilité dans un processus d'abstraction spatiale concomitant. Ce texte se termina par quelques réflexions sur l'actualité d'une telle théorie de la spatio-temporalité abstraite pour étudier l'actuelle financiarisation du capital. 


\section{Henri Lefebvre, l'espace et la médiation spatiale abstraite du capitalisme L'alternative lefebvrienne au fétichisme de l'espace}

Chaque société (donc chaque mode de production avec les diversités qu'il englobe, les sociétés particulières où se reconnaît le concept général) produit un espace, le $\operatorname{sien}^{7}$.

Cette déclaration bien connue d'Henri Lefebvre exprime en condensé les principes fondateurs de la théorie de l'espace qu'il a développée à partir des années 1960. Cette théorie est née du désir de formuler une conception alternative de la spatialité qui échapperait à la tendance dominante - et vivement dénoncée par Lefebvre - au «fétichisme de l'espace». Contre la géométrie euclidienne et la physique newtonienne, il a d'abord voulu affirmer que l'espace ne pouvait être réduit à un objet neutre et sans vie. L'espace n'est pas seulement un fait de la nature qui ne se manifeste que par les propriétés physiques mesurables qu'il présente à l'examen empirique, mais un produit de la société. Il est une création de l'activité humaine qui «incorpore des actes sociaux, ceux de sujets à la fois collectifs et individuels, qui naissent et meurent, pâtissent et agissent ${ }^{8}{ }^{\prime}$. En somme, l'espace est modelé à l'image des rapports qui constituent la société. Tantôt conflictuels, tantôt reproductifs, ces rapports ne se déploient pas seulement dans des pratiques observables relevant, selon Lefebvre, du domaine du «perçu». La production de l'espace se nourrit tout autant de représentations (du «conçu») que de l'expérience quotidienne façonnée par ces pratiques imprégnées de symbolisme (du «vécu»). La spatialité propre à chaque société résulte ainsi de l'interaction entre ces dimensions de l'existence humaine qui se distinguent les unes des autres par le rapport particulier à l'espace auquel elles renvoient: le perçu prend forme dans des pratiques spatiales, le conçu dans des représentations de l'espace et le vécu dans des espaces de représentations?.

La célèbre affirmation de Lefebvre suppose également que l'espace soit beaucoup plus que le résultat d'un processus qui lui serait antérieur et plus fondamental. En produisant de la spatialité, chaque société ne donne pas naissance à n'importe quelle forme d'espace. Elle crée, nous dit Lefebvre, «le sien", celui qui lui correspond, qui en constitue l'un des traits distinctifs et qui lui permet de se maintenir dans le temps sous cette forme spécifique.

7. H. Lefebvre, La production..., op. cit., p. 40.

8. Ibid., p. 43.

9. Pour être entièrement fidèle à la démarche et à la pensée de Lefebvre, il aurait fallu que la discussion sur la spatialité du capitalisme présentée plus loin dans ce texte tienne compte de ces trois dimensions de la théorie lefebvrienne de l'espace. Par manque d'espace et parce que je souhaite d'abord insister sur le procès d'abstraction que l'on retrouve au cœur de la production de l'espace dans le capitalisme, ce texte mettra surtout l'accent sur les pratiques spatiales constitutives de cette abstraction. 
L'espace intervient alors dans le processus social qui fait de la société ce qu'elle est. Il n'en est pas le simple reflet passif. Lefebvre dira de la spatialité qu'elle est à la fois produit et production (ou force productive), de telle sorte qu'une fois créée, elle devient le support essentiel des pratiques et représentations qui entreront dans la production ultérieure de l'espace, que ce soit pour le reproduire ou le transformer : «le concept d'espace social se développe donc en s'élargissant. Il s'introduit au sein du concept de production et même l'envahit; il en devient le contenu, peut-être essentiel ${ }^{10}{ }^{\prime}$. L'espace est donc une médiation fondamentale de l'activité sociale et, à ce titre, il peut également être source d'historicité et de mouvement.

Concevoir l'espace à la manière d'une médiation sociale permet aussi de reconsidérer la thèse de la secondarité de l'espace par rapport au temps. Comme nous l'avons vu, cette dernière présente la spatialité comme la dimension statique, figée, anhistorique et apolitique de l'existence humaine et l'oppose à une temporalité par nature dynamique constituant la véritable source du possible. En soutenant la thèse d'une médiation par l'espace, Lefebvre a, à l'inverse, voulu démontrer que la spatialité participait à l'Histoire au même titre que le temps. Si sa théorie conteste la priorité accordée au temps, elle n'en est pas non plus une de la primauté du spatial. Elle vise plutôt à affirmer l'interdépendance du temps et de l'espace en tant que catégories coconstitutives de la réalité et en tant que dimensions fondamentales de la pratique à travers lesquelles l'Histoire se réalise.

Sans remettre en cause le fait que l'espace puisse être source de fixité, Lefebvre propose d'envisager cette propriété comme une condition de possibilité du dynamisme temporel et, de manière générale, comme le support essentiel du mouvement historique ${ }^{11}$. Pour lui c'est «le passage incessant de la temporalité (succession, enchaînement) à la spatialité (simultanéité, synchronisation)» qui «définit toute activité productrice ${ }^{12}$. Comme l'a récemment fait remarquer le géographe Noël Castree au sujet de l'accumulation capitaliste, celle-ci serait évanescente et sans pouvoir réel si elle ne produisait pas d'espace. En revanche, cette spatialité serait elle-même inconcevable abstraction faite de la nécessité qu'elle trouve dans une temporalité historiquement spécifique ${ }^{13}$.

10. H. Lefebvre, La production..., op cit., p. 102-103.

11. Chez Lefebvre, l'espace n'est pas uniquement fixité. Il peut également être source de mouvement. David Harvey a repris cette idée dans son étude de la géographie du capital. Celle-ci est, selon lui, le produit des exigences contradictoires de fixité et de mobilité que rencontre le capital à toutes les étapes de son procès d'accumulation. Harvey expose les fondements de cette dialectique spatiale opposant fixité et mobilité dans The Limits to Capital, Chicago, The University of Chicago Press, 1982, 478 p.

12. Ibid., p. 87.

13. Noel Castree «The Spatio-temporality of Capitalism». Time and Society, vol. 18, n 1, 2009, p. 27. 


\section{La dialectique spatiale abstraite-concrète de la reproduction du capitalisme}

Les deux thèses centrales de la théorie de Lefebvre sur l'espace (en tant que produit historiquement déterminé et médiation sociale) sont le fruit d'une longue réflexion qu'il a menée au fil de ses nombreuses recherches sur la ville, la vie rurale, l'habitation de banlieue et l'architecture ${ }^{14}$. Ces travaux lui ont non seulement inspiré sa conception de l'espace en général, mais aussi sa compréhension de la logique spécifique présidant à la production de l'espace dans les sociétés capitalistes. Lefebvre était un lecteur attentif de Marx et l'analyse qu'il a développée de la spatialité propre au capitalisme a été fortement influencée par la critique du capital formulée dans les Grundrisse et Le Capital. C'est pourquoi, avant d'aller plus loin dans cet exposé des thèses de Lefebvre sur l'espace, il m'apparaît nécessaire de revenir brièvement sur certaines idées maîtresses de la critique marxienne du capitalisme.

La critique de Marx est celle d'un monde qui se présente comme une «immense accumulation de marchandises ${ }^{15}$ », où les êtres humains ne consomment plus directement les fruits de leur labeur, mais produisent des marchandises qui n'ont pour eux d'autre utilité que de servir de moyens d'échange pour l'obtention des fruits du travail des autres. Les marchandises sont à la fois valeurs d'échange pour leur producteur et valeurs d'usage pour ceux qui se les procurent. Dans ce type de société, l'activité concrète créatrice de valeurs d'usage est assujettie au principe dominant de l'échange en vertu duquel les choses n'ont de valeur qu'en tant qu'elles peuvent être vendues sur un marché. Cette production en vue de l'échange n'est possible qu'au prix d'une subordination de la finalité qualitative et particulière à chaque activité au critère commun que partagent tous les produits de tous les travaux, celui de représenter une dépense quantitative d'énergie humaine. Les valeurs d'usage ne deviennent valeurs d'échange que lorsque le travail singulier qui les constitue est converti en travail abstrait vidé de son contenu et cesse d'être le moyen d'une fin substantielle pour apparaitre comme le moment du procès sans fin de «la production pour la production». Le capital est ainsi une forme sociale qui se développe sous l'impulsion d'un procès d'abstraction qui s'approprie l'activité humaine pour la transformer en travail en général.

Le travail abstrait créateur de valeur est aussi, chez Marx, doté d'une forte composante temporelle. En effet, la grandeur de la valeur est fonction de la quantité de travail abstrait que contient une marchandise qui, elle-

\footnotetext{
14. Dans un ouvrage intitulé Henri Lefebvre on Space, Lukasz Stanek retrace l'évolution de la pensée spatiale de Lefebvre à travers les travaux qu'il a réalisés avec différents collaborateurs ainsi que les débats qui l'ont opposé à plusieurs personnalités de la sociologie et de l'architecture françaises. Lukasz Stanek, Henri Lefebvre on Space. Architecture, Urban Research, and the Production of Theory, Saint-Paul, University of Minnesota Press, 2011, 369 p.

15. Karl Marx, Le Capital, Paris, Garnier-Flamarion, 1867/1969, 696 p.
} 
même, se mesure de manière objective par sa durée. Les marchandises se distinguent les unes des autres non plus d'abord par leurs qualités intrinsèques, mais par la quantité plus ou moins grande de temps de travail qu'elles ont absorbée. La valeur de chacune dépendra alors de la durée du travail abstrait engagé dans leur production et du rapport de celle-ci au temps moyen requis pour créer les marchandises nécessaires à la reproduction de la force de travail, que Marx nomme le temps de travail socialement nécessaire.

Dans Temps, travail et domination sociale, Moishe Postone a poursuivi cette analyse de la valeur en précisant que son pouvoir de régulation reposait sur des pratiques et un rapport au temps qui étaient eux-mêmes spécifiques au capitalisme. Le temps de travail socialement nécessaire n'exprime pas n'importe quel type de temporalité, mais un temps abstrait et dépouillé de son contenu historique. Cette mesure de l'activité se présente sous une forme homogène, continue et indépendante des circonstances de la vie (les secondes, les heures, etc. $)^{16}$. Elle s'oppose au temps concret de l'expérience vécue qui est lié «aux périodicités de la vie humaine» et aux «processus particuliers» qui la composent ${ }^{17}$. Postone souligne que la temporalité historique concrète ne s'efface jamais entièrement devant son Autre abstrait, mais qu'elle est plutôt reconstituée en permanence par cette dernière sous la forme inchangée d'un présent perpétuel qui s'impose comme norme de productivité.

Dans ses travaux sur l'espace, La production de l'espace notamment, Lefebvre reprend la théorie marxienne de l'abstraction capitaliste pour en développer davantage la dimension spatiale qui, comme nous le verrons sous peu, est loin d'être absente chez Marx, mais qui n'y est toutefois pas formellement explicitée. En effet, si le capitalisme se distingue des formes de sociétés qui l'ont précédé par le rapport abstrait au temps et au travail qu'il institue, Lefebvre soutient qu'il puise également son pouvoir de domination dans une spatialité soumise à un procès d'abstraction similaire. Selon lui, le principal effet du capital sur l'espace est de lui donner la forme d'une marchandise qui, comme n'importe quelle autre, est doublement constituée d'une dimension concrète et d'une dimension abstraite. La première, que Lefebvre nomme l'espace social (plutôt que concret), renferme la valeur d'usage de l'espacemarchandise (son utilité qualitative), tandis que la seconde, l'espace abstrait, exprime sa valeur d'échange.

D'une manière semblable aux deux formes du travail constitutif de la marchandise chez Marx, le social et l'abstrait sont, pour Lefebvre, les deux facettes d'un même espace et sont inséparables l'une de l'autre, bien que hiérarchi-

\footnotetext{
16. Moishe Postone, Temps, travail et domination sociale. Une réinterprétation de la théorie critique de Marx, Paris, Éditions Mille et une nuits, 2009, p. 300.

17. Ibid., p. 298.
} 
quement mises en relation. L'espace abstrait est la négation de l'espace social qui ne peut cependant exister sans le support qu'il trouve dans ce dernier. Il constitue l'espace en général, quantitatif et dépouillé de tous les usages qualitativement distincts dont son vis-à-vis social est la manifestation. Dans cette spatialité abstraite, le «vécu s'écrase ${ }^{18}$ ». Elle se conçoit «en dehors de tout contenu défini» et par la négation de tout «ce qui [la] précède et [la] supporte: l'historique, le religieux-politique ${ }^{19}{ }^{»}$. L'abstrait se construit ainsi dans une opposition constante à l'espace social qui est, quant à lui, produit de la vie quotidienne et de l'interaction de tous les membres de la sociétéro ${ }^{20}$.

Bien qu'elle remplisse une fonction centrale dans la reproduction des sociétés capitalistes, cette forme abstraite de spatialité n'est pas le fruit de l'histoire récente. Son existence est antérieure à l'avènement du capital. Selon Lefebvre, elle tire son origine de la géométrie euclidienne et du droit de propriété romain. Toutefois, ce qui fait la spécificité du capitalisme est la place désormais dominante et structurante occupée par la spatialité abstraite dans ce type de société. L'abstraction spatiale y acquiert une «existence sociale» qui, comme le travail abstrait de Marx, n'a rien d'une opération mentale. Elle est, au contraire, dotée d'un pouvoir «réel» de détermination sur l'activité sociale. Ce pouvoir se manifeste plus particulièrement dans la capacité historiquement nouvelle de la composante abstraite de l'espace à «absorber l'usage» et à le reconstituer sous une forme plus adaptée aux exigences de l'échange marchand ${ }^{21}$. La production de l'espace proprement capitaliste prend ainsi la forme d'un procès d'abstraction qui se subordonne et transforme en permanence la composante sociale et historique de l'espace. Cependant, comme l'abstrait et le social sont les deux facettes inséparables du même espace (capitaliste), le premier ne peut jamais dominer le second au point d'en entraîner la disparition complète. Il se priverait du même coup de son contenu essentiel et mettrait fin au mouvement qui lui donne vie. C'est pour cette raison que, suivant de près le raisonnement de Marx, Lefebvre dira de cette abstraction qu'elle est un procès, une "abstraction en acte", qui «ne peut se concevoir abstraitement» sans référence au contenu qu'elle «saisit dans une pratique qui le traite $»^{22}$.

La thèse de la soumission de l'espace capitaliste à une dialectique abstraite-concrète permet également de rapprocher les deux positions philoso-

18. H. Lefebvre, La production..., op cit., p. 63.

19. Ibid., p. 62-63 et 120 .

20. Cette définition de l'espace social tel qu'il est conçu par Lefebvre est largement inspirée de celle qu'en donne Mark Gottdiener dans «A Marx of our Time : Henri Lefebvre and the Production of Space», Sociological Theory, vol. 11, n 1, 1983, p. 131.

21. H. Lefebvre, La production..., op cit., p. 354

22. Ibid., p. 309 et 352. 
phiques, en apparence diamétralement opposées, consistant à concevoir l'espace soit comme le domaine statique de l'être soit comme celui du devenir. Chez Lefebvre, la spatialité intervenant dans la reproduction du capital n'est pas exclusivement l'un ou l'autre, mais les deux. Sous l'emprise du capital, l'espace existe effectivement sous une forme sociale (historique) ouverte au mouvement et à la différence, dont l'expression est cependant captée et modelée en permanence par une spatialité abstraite qui transforme ce potentiel de changement en un moment de pure reproduction ou, pour reprendre l'expression de Moishe Postone, en un présent perpétuel. Sous cette forme, l'espace a véritablement le pouvoir de nier le temps historique du devenir, tout autant d'ailleurs que l'espace social dans lequel cette temporalité prend forme.

L'abstraction spatiale procède, selon Lefebvre, de trois manières qui contribuent à donner à l'espace une forme plus adéquate à la reproduction du capital: par homogénéisation, fragmentation et hiérarchisation. L'homogénéisation est le processus par lequel l'espace est rendu uniforme et vidé de sa substance sociale. Elle réduit celui-ci à ses propriétés quantitatives et mesurables pour en faire une chose échangeable parmi les autres, une marchandise, qui «prend ainsi la réalité autonome (en apparence) [...] de l'argent ${ }^{23}$ ». Il en résulte «un espace mortel» qui tue ses conditions (historiques), ses propres différences (internes), les différences (éventuelles), pour imposer l'homogénéité abstraite » ${ }^{24}$. L'espace n'est alors plus un lieu d'interactions et de culture, mais un simple contenant vide se présentant «naturellement» sous la forme fétichisée de la propriété découpée en lots et autres unités de valorisation. En effet, il ne suffit pas, pour rendre échangeable l'espace devenu marchandise, de l'homogénéiser. Il faut également que cette uniformisation quantitative permette la comparaison et qu'elle serve de base à une subdivision nouvelle de l'espace en unités qui ne présentent à l'évaluation que des différences de grandeur.

La seconde étape du procès spatial d'abstraction, la fragmentation, produit ces «cellules quasiment identiques ${ }^{25}$ » qui ne se distinguent que par leurs propriétés abstraites. C'est ainsi, par exemple, que, dans un régime de propriété privée du sol, les caractéristiques de l'espace qui priment pardessus tout sont celles qui sont données par des calculs de superficie, de valeur au mètre carré, les divisions cadastrales et les règles municipales en matière de zonage et d'évaluation foncière. La fragmentation spatiale quantitative fournit également le cadre nouveau dans lequel le social jusqu'ici

23. Ibid., p. 388.

24. Ibid., p. 427.

25. Ibid., p. 389. 
réprimé peut s'exprimer, mais sous une forme qui lui est dictée par le procès d'abstraction, en tant que condition de la valorisation différenciée de chaque espace. La subdivision de l'espace produit alors de nouveaux agencements et rapports entre des unités spatiales homogènes. Elle crée une hiérarchisation nouvelle des espaces qui met en évidence leurs distinctions quantitatives et qui confirme la prédominance propre aux sociétés capitalistes de l'abstrait sur le concret.

\section{La spatialité et son rapport au temps dans l'analyse marxienne du développement du capitalisme}

La filiation marxienne de la théorie lefebvrienne de la production de l'espace capitaliste ne se limite pas seulement au fait que son auteur ait tenté d'élargir le champ d'application du procès d'abstraction constitutif du capital au domaine de la spatialité. Comme l'a bien démontré Franck Fischbach dans La privation de monde, Marx avait déjà établi, dans certains passages du Capital et des Grundrisse, un rapport essentiel entre le capital et l'espace $^{26}$. On retrouve entre autres dans ces deux ouvrages une analyse historique de la formation d'une spatialité nouvelle, sur laquelle Marx fonde son étude de l'émergence et du développement ultérieur du capital, et qui est en plusieurs points semblables à l'espace abstrait décrit par Lefebvre. La nouveauté de cet espace se manifeste, dans un premier temps, dans la rupture radicale qu'il opère avec le mode d'occupation traditionnel (pré-capitaliste) $\mathrm{du}$ territoire puis, avec la diffusion de la grande industrie et du machinisme dans un second temps, dans son affirmation comme puissance autonome (un capital fixe) qui accroît la dépendance de la société dans son ensemble à la contrainte du travail abstrait. Ainsi, bien que la question spatiale n'ait pas été problématisée de manière explicite par Marx, elle apparaît en toile de fond de sa présentation de l'évolution du capitalisme en tant que support essentiel au développement de ce dernier et qui, pour y jouer ce rôle, a dû se couper (s'abstraire) de son passé.

Pour étayer cette hypothèse, je reviendrai brièvement sur les passages des travaux de Marx où il expose les conditions historiques des deux stades de développement du capital, ceux de la subordination formelle et de la subordination réelle du travail au capital. À chacun de ces moments, il sera possible de constater qu'il y avait déjà chez Marx l'intuition d'une dialectique spatiale participant, de manière fondamentale et inséparable de la temporalité, à la réalisation du processus d'abstraction capitaliste.

26. Franck Fischbach, La privation de monde. Temps, espace et capital, Paris, Librairie Philosophique J. Vrin, coll. «Problèmes et controverses», 2011, p. 109. 


\section{Les conditions spatiales de l'avènement du capitalisme. La subordination formelle de l'espace au capital}

Dans la huitième et dernière section du Capital, Marx a cherché à établir les conditions de l'accumulation primitive qui représente pour lui le «point de départ» historique de la production capitaliste et qui lui permet d'expliquer l'origine de celle-ci sans déjà présupposer son existence. Le sens qu'il donne à ce concept est bien connu: les conditions propices à la naissance du capitalisme ont été réunies au terme de transformations historiques qui ont «fait divorcer le travail d'avec ses conditions extérieures» et par lesquels les moyens de production ont été «arrachés sans phase aux producteurs qui les employaient à réaliser leur propre travail $»^{27}$. Avant toute chose, ce divorce a été réalisé par la séparation du travailleur de la terre, qui en était jusque-là «le laboratoire naturel», pour en faire la propriété d'autrui ${ }^{28}$. Il y a donc, à l'évidence, une condition spatiale à l'accumulation primitive, ce qui suggère que l'avènement du capitalisme aurait été médiatisé par la production d'un espace nouveau sans lequel cette révolution historique n'aurait pu être envisageable. Marx s'est surtout intéressé à l'exemple de l'Angleterre où les premières bases de la production capitaliste ont été jetées à la suite d'une succession de modifications apportées au régime foncier féodal: conversion des terres arables en pacages, démolition des maisons et cottages paysans, spoliation des biens de l'Église catholique, abolition par les Stuart de la constitution féodale du sol, expropriation des champs communaux (les enclosures) et «éclaircissement des biens fonds» (clearing of estates). Ces transformations, dont il n'offre qu'une présentation sommaire au chapitre XXVII du Capital (L'expropriation de la population campagnarde), ont, pour lui, toutes concouru à la formation de la propriété foncière moderne (capitaliste), c'està-dire la propriété immobilière organisée en marché.

Le propre de ce nouveau régime foncier est d'instituer une barrière monétaire entre les individus et leur accès à la terre. Il crée aussi un droit exclusif sur les usages et les fruits de celle-ci pour celui qui en fait l'acquisition au moyen de l'argent. Ce régime interdit le libre accès à la terre et met fin, par la même occasion, au mode pré-capitaliste d'occupation du territoire qui, au-delà des diverses formes historiques et régionales qu'il ait pu prendre (dans la collectivité tribale, la communauté asiatique ou encore la propriété féodale, par exemple), reposait selon Marx sur deux principes invariables: un rapport de l'homme à ses conditions de production «en tant qu'elles sont les siennes, qu'elles sont présupposées avec sa propre existence» et un rapport

27. K. Marx, op cit., p. 528.

28. Karl Marx, Manuscrits de 1857-58 dits «Grundrisse», Paris, Les Éditions sociales, 1857-58/2011, p. 432 et 462. 
à ces moyens du travail qui est «médiatisé par l'appartenance à une communauté historiquement développée ${ }^{29}$.

La propriété privée de la terre transforme radicalement ces deux conditions. D'une part, elle dissout la propriété immédiate de l'instrument de travail. Elle fait de celui-ci une chose étrangère au travailleur qui ne lui est plus simplement donnée. D'autre part, la constitution d'un marché foncier, et plus particulièrement la possibilité de vendre le sol, désagrègent le lien qui rendait possible l'utilisation ancienne de la terre. Elle substitue à la coutume et aux relations personnelles de domination féodales qui liaient autrefois les individus à leur communauté un rapport nouveau au territoire qui prend la forme d'un paiement monétaire impersonnel et indifférent au statut des personnes qu'il met en relation.

L'espace produit de la dissolution de la relation traditionnelle entre l'individu, la communauté et les conditions objectives du travail est, en d'autres termes, l'espace abstrait dont Lefebvre a fait le concept central de sa théorie de la spatialité capitaliste. Cette espace est abstrait dans la mesure où il se manifeste en tout premier lieu dans l'uniformité de la contrainte monétaire qu'il impose à quiconque veut y avoir accès et parce que la reproduction de cette obligation se présente comme le principe déterminant de tout usage que l'on peut en faire. La spatialité homogène fractionnée en unités commercialisables de superficie et de valeur différentes a pour effet, selon l'expression de Engels, «de trancher le cordon ombilical qui rattachait au sol le travailleur du passé ${ }^{30} »$ et de vider l'espace des rapports corporatifs et de clientèle dans lesquels «prédominait la valeur d'usage, la production pour l'usage immé$\operatorname{diat}^{31}$ ». Pour Marx, une fois réduit à «une forme purement économique » et abstraite de «tous ses enjolivements et ses amalgames politiques et sociaux d'autrefois ", l'espace produit par la propriété foncière moderne représente l'un «des plus grands achèvements du système capitaliste », puisque c'est en elle que se crée l'obligation au travail salarié, la condition fondamentale qui permet au capital de voir le jour et de se renouveler sans cesse ${ }^{32}$.

En effet, c'est dans un tel cadre spatial qu'apparait la figure du travailleur «sans feu ni lieu», qui n'a pour seule possession et seul lien possible avec l'ensemble de la société le temps de travail qu'il doit désormais vendre pour assurer sa subsistance. La propriété foncière fournit ainsi l'assise spatiale de la soumission formelle du travail au capital fondée sur l'extraction d'une plus-

29. K. Marx, Le capital, op cit., p. 443-451.

30. Friedrich Engels, La question du logement, Paris, Éditions sociales, 1976, p. 33.

31. K. Marx, Le capital, op. cit., p. 462.

32. Karl Marx, «Le capital. Livre troisième». Dans CEuvres.Économie II, Paris, La pléiade, 1864-75/1968, p. 1288-1289. 
value absolue qui représente, chez Marx, la forme initiale de l'exploitation capitaliste.

Au stade de la soumission formelle, le capital s'approprie l'activité sociale productive sans toutefois en modifier le contenu. Marx précise qu'il «n'existe aucune innovation dans le mode de production lui-même: le procès de travail se déroule exactement de la même manière qu'autrefois, hormis qu'il est maintenant subordonné au capital ${ }^{33}{ }^{»}$. Ici, le capital se nourrit de forces sociales telles qu'elles se présentent à lui, c'est-à-dire telles qu'elles ont été historiquement constituées dans un contexte pré-capitaliste. En l'absence de moyens de réguler la manière dont s'accomplit l'activité productive, le pouvoir du capital sur les travailleurs réside dans sa capacité à rendre leur travail obligatoire et d'en fixer la durée. L'enjeu de la soumission formelle est donc l'extension de la journée de travail au-delà du temps socialement requis pour la reproduction de la force de travail. La plus-value absolue, caractéristique de cette première phase de développement du capitalisme, résulte dès lors du surtravail réalisé au cours de ce temps supplémentaire où le travailleur n'a œuvré que pour le capital.

À ce moment précis, l'espace productif se présente sous une double forme: en tant que propriété du capital, qui fonde l'obligation pour le travailleur de soumettre son activité à la temporalité homogène du procès de production capitaliste (la journée de travail), et, simultanément, en tant que support à la reproduction d'un savoir productif et d'une culture qui ne doivent rien au capital. La plus-value absolue est par conséquent le fruit du rapport asymétrique qu'entretiennent les deux composantes de ce même espace, en vertu duquel l'espace abstrait de la propriété privée saisit l'espace social de la pratique productive traditionnelle et lui prescrit une durée fixée par l'exigence, elle-même abstraite, du temps de travail socialement nécessaire. L'assujettissement de la spatialité concrète-sociale à son opposé abstrait constitue, en ce sens, la condition préalable et nécessaire (la médiation) d'un mode d'exploitation du travail reposant sur le prolongement du temps de travail. Ce n'est que sous l'effet d'une telle dynamique spatiale que le temps abstrait de la production, dans son opposition à la temporalité variable et qualitative inhérente à toute pratique, peut s'ériger en norme structurante de l'activité sociale.

Cependant, on ne pourrait pour autant conclure de ces explications que le capitalisme est un mode de production dans lequel la spatialité prime sur la temporalité. Si l'espace abstrait pose effectivement les conditions de l'action

33. Karl Marx, Un chapitre inédit du capital. Premier livre. Le procès de production, 1867, p. 83. En ligne: www.uqac. uquebec.ca/zone30/Classiques_des_sciences_sociales/index.html. 
de la norme temporelle abstraite (le temps de travail socialement nécessaire), cette dernière demeure le moteur de la production capitaliste et constitue, à ce titre, le principal incitatif à abstraire l'espace de sa substance sociale et historique. Quel intérêt y aurait-il alors à étendre l'abstraction spatiale et à en faire le mode dominant d'occupation du territoire si ce n'était précisément des perspectives de revenus offertes par la possibilité de dégager de cet espace-marchandise du temps de surtravail ? Ce rapport particulier de l'espace au temps n'a pas seulement été fondamental dans la production des conditions extérieures au capital de l'accumulation primitive, mais aussi dans l'évolution du capitalisme sous sa forme que Marx jugeait la plus développée, celle de la soumission réelle du travail au capital.

\section{Le capital fixe et la soumission réelle de l'espace au capital}

La principale distinction que Marx établit entre la soumission formelle et la soumission réelle est d'ordre temporel. Cette seconde forme de l'exploitation capitaliste voit le jour dans l'impossibilité d'allonger indéfiniment la journée de travail afin de soutirer aux salariés davantage de surtravail. Ce problème sera progressivement compensé par une redéfinition de l'organisation du travail visant à le rendre plus productif, c'est-à-dire capable de produire plus de marchandises dans l'intervalle de temps fixe donné par la journée de travail. La mise en place de nouvelles techniques de travail (la subdivision de l'activité productive en tâches simples et répétitives, par exemple) permettra d'abaisser le temps de production des marchandises et, par conséquent, de diminuer le temps de travail socialement nécessaire à la reproduction de la force de travail, c'est-à-dire la norme sociale de production qui permet de départager le temps au cours duquel l'ouvrier travaille pour lui de celui qu'il consacre exclusivement au capital. Il se crée dans ces conditions un nouveau type de plus-value qui prend forme dans la part croissante de temps de travail que le capital soutire gratuitement aux travailleurs sans avoir à modifier la durée de la journée pendant laquelle ils sont à son emploi. Marx explique que cette plus-value n'est plus absolue, mais relative, "parce que s'est accru non pas le quantum de travail absolu, mais [que] c'est le quantum de travail nécessaire qui a diminué et [que], de ce fait, s'est accru le surtravail relatif ${ }^{34}{ }^{\text {» }}$.

Alors que, dans le premier cas, le capital s'appropriait l'activité productive telle qu'elle se présentait à lui, dans le second, le surplus de valeur résulte d'une transformation et d'une reconfiguration du contenu même du travail. Ainsi, le passage à la soumission réelle du travail au capital engage-t-il une destruction des métiers traditionnels et la conversion des travailleurs en pro-

34. K. Marx, «Le capital. Livre troisième»..., op. cit., p. 304. 
duits intrinsèques du capital, qui ne sont désormais que de simples rouages d'un procès de production qui leur est entièrement extérieur. Le capital n'impose plus seulement une durée au travail, mais s'érige en "force étrangère et hostile» qui a également la capacité de lui prescrire un contenu ${ }^{35}$.

Marx ne s'est pas contenté d'exposer de manière formelle ce qui distingue ce second mode de soumission au capital du premier. Il s'est aussi intéressé, comme il l'a fait dans le cadre de son analyse de l'avènement du capitalisme, aux conditions historiques du passage de l'un à l'autre. Une fois de plus, on remarque que c'est par un processus de transformation spatiale, plus précisément par une modification de l'organisation du travail dans l'espace productif, que la soumission réelle succède à la soumission formelle. Ce processus trouve sa possibilité dans la spatialité qui se présente à lui, c'est-à-dire dans l'espace abstrait historiquement constitué par l'accumulation primitive et qui crée une masse de travailleurs libres et disponibles à être employés. Cet espace fournit les conditions de la généralisation d'une production à grande échelle fondée sur la capacité dont dispose désormais le capital d'employer simultanément un grand nombre de travailleurs en un même lieu.

Marx a consacré les chapitres XIII à XV du Capital à l'étude des conséquences sur l'activité productive de ce phénomène de massification du travail. Il s'est d'abord penché de manière générale sur les rapports de coopération qui se développent dans ce nouveau contexte productif, avant d'analyser leur évolution particulière dans la manufacture et dans la grande industrie. La manufacture se distingue de la coopération simple, qui se forme lorsque des individus réunis sous un même toit s'adonnent à la même activité, en introduisant une division technique et sociale du travail artisanal qui le rend encore plus productif. L'industrie franchit quant à elle un pas de plus vers la soumission réelle du travail au capital en imposant le machinisme comme principal moteur de la production. Elle se démarque des autres modes d'exploitation du travail massifié par son pouvoir d'objectivation du savoir et de l'habileté humaine passés sous la forme de machines, infrastructures et procédés scientifiques appartenant en exclusivité au capital. Les fruits du travail passé deviennent ainsi capital fixe, immobilisé dans l'espace productif, qui, parce qu'il n'appartient plus au travailleur, peut désormais lui être imposé comme condition de son activité. Le plus grand accomplissement de la production industrielle réside, selon Marx, dans l'avènement du capital fixe qui se substitue tendanciellement au travail vivant comme organe principal de la création de marchandises et qui contraint les travailleurs à s'ajuster à son fonctionnement :

35. K. Marx, Un chapitre inédit du capital..., op. cit. , p. 82. 
dans le capital fixe, le moyen de travail, sous son aspect matériel, $[\ldots]$ se présente matériellement face à l'ouvrier comme capital. Le savoir apparait dans la machinerie comme quelque chose d'étranger à l'ouvrier; et le travail vivant apparaît subsumé sous le travail objectivé agissant de façon autonome. Et dans la mesure où son action n'est pas conditionnée par le besoin [du capital], l'ouvrier apparait comme superflu ${ }^{36}$.

En ce sens, la grande industrie dominée par le capital fixe procède à une inversion fondamentale du procès de travail par laquelle les sujets de la production autrefois producteurs indépendants deviennent les objets d'un procès entièrement déterminé par ce qui en constituait auparavant les moyens. Les travailleurs se muent alors en "pièces du système » composé par une accumulation de forces productives appropriées par le capital et qui se dressent devant eux comme un "organisme [leur] imposant sa violence ${ }^{37}$. C'est dans ce rapport de dépendance complète du travailleur qui s'étend maintenant jusqu'au contenu de son activité que le capital trouve les moyens de soumettre celui-ci à ses exigences d'accroissement de la productivité. C'est donc aussi dans ce mode particulier de spatialisation du procès productif placé sous l'égide du capital fixe que l'accumulation capitaliste revêt sa «forme la plus adéquate» en ce qu'elle permet de cette manière à la production de «reproduire ses propres conditions ${ }^{38}$.

L'accession du capital à une forme plus développée sous laquelle il acquiert les moyens d'assurer lui-même son expansion dépend ainsi de la production d'une spatialité qui s'impose au travail en tant que force qui lui est totalement étrangère et qui tend à dissoudre encore davantage l'ancrage de cette activité dans un passé pré-capitaliste. En plus d'insister sur le caractère abstrait de cet espace qui assure le passage de la soumission formelle à la soumission réelle au capital, il est important de souligner que, à la lumière de l'analyse proposée par Marx dans les Grundrisse, il n'aurait pu voir le jour autrement que par la médiation de la spatialité déjà abstraite de l'accumulation primitive. Marx précise que «la machine n'entre en jeu que là où la puissance de travail existe en masse» et que lorsque «le travailleur est placé vis-à-vis d'elle dans un rapport d'ouvrier salarié ${ }^{39}$.

Cet espace qui prive le travailleur d'un accès direct à ses moyens de travail est la condition concrète et préalable de l'appropriation subséquente par le capital du travail et de la connaissance passés sous la forme d'un capital fixe capable de dicter au travailleur la nature et le rythme de son activité. Le capital fixe puise dans cet état antérieur de privation la possibilité de libérer le

36. K. Marx, «Le capital. Livre troisième»..., op cit., p. 655

37. Ibid., p. 653.

38. Ibid., p. 654 et 682.

39. Ibid., p. 658 
procès de production capitaliste de sa dépendance à l'égard du principal facteur qui lui était encore extérieur, à savoir le contrôle des ouvriers sur l'acte productif. En somme, le machinisme trouve dans les conditions spatiales abstraites de la subordination formelle la nouvelle concrétude lui permettant de donner naissance à un espace encore plus abstrait qui fait de la sphère productive un lieu totalement étranger au travailleur.

Pour reprendre la terminologie de Lefebvre, l'espace de production est plus homogène qu'il ne l'avait été jusqu'ici dans la mesure où il est totalement indifférent quant à l'identité particulière et à l'histoire dont sont porteurs les individus qu'il emploie. Ceux-ci ne représentent pour lui, en tout premier lieu, qu'une capacité de travail à mettre en œuvre selon des critères et principes qui ne sont pas de leur ressort. Ce développement de l'abstraction spatiale par homogénéisation s'accompagne aussi d'une nouvelle fragmentation hiérarchisée de l'espace en unités qui ne se distinguent plus seulement par leurs différences quantitatives de valeur, mais également par leur degré différencié de productivité.

L'espace «réellement» subordonné au capital ne se limite pas à arracher l'activité sociale à toute détermination extérieure à la logique d'accumulation capitaliste. Il recompose celle-ci pour lui donner une nouvelle forme concrète. L'espace de la grande industrie n'est pas seulement le lieu d'expression de grandeurs quantitatives, mais il permet aussi de nouveaux usages dans lesquels prennent forme les différences de productivité qui sont à l'origine de la plus-value relative. Ainsi, même au stade plus avancé de la subordination réelle, le capital demeure intrinsèquement dépendant de l'existence d'une activité concrète qui est pour lui source de travail abstrait. L'abstraction capitaliste ne parvient jamais à éviter une certaine appropriation de l'espace productif par le travail. Il s'agit d'ailleurs d'une contradiction à laquelle les spécialistes en management et en organisation du travail sont quotidiennement confrontés. L'espace est certes soumis à la domination abstraite du capital, mais il n'est jamais pur statisme ou unique force opposée au devenir. Il est, comme aimait le rappeler Lefebvre, source de contradiction, de conflits et donc aussi de transformation.

\section{Une domination du temps par l'espace?}

La lecture présentée ici des travaux de Marx permet de considérer la création d'un espace productif dominé par la figure abstraite du capital fixe comme l'exigence du procès temporel de soumission réelle du travail au capital. De ce point de vue, aucun changement dans le rythme et la forme du travail (sa productivité) ne serait envisageable sans une modification correspondante 
de l'espace qui lui sert de support (de médiation) et qui permet l'accomplissement des gestes par lesquels cette activité se réalise. Ce n'est que dans un cadre spatial particulier, où le capital fixe s'érige en puissance autonome et opposée au travailleur, que le capital acquiert une capacité de détermination sur le contenu de l'activité productive et qu'il parvient à imposer une nouvelle temporalité à l'exécution de cette dernière. Dans Histoire et conscience de classe, Georg Lukàcs a fait de cette idée l'un des fondements de son concept de réification. Selon lui, l'existence réifiée est celle de l'homme qui a été réduit à n'être qu'une "partie mécanisée» d'un «système achevé et clos » qui agit «sans l'influence possible d'une activité humaine». Le procès de réification fait également intervenir l'espace d'une manière toute particulière. Il n'est possible que dans la mesure où le temps est soumis à un espace qui le prive de tout son dynamisme et qui en fait une dimension de l'existence entièrement inféodée aux exigences spatiales du capital. Plus précisément, Lukàcs dira que la réification a pour effet de ramener "le temps au niveau de l'espace ${ }^{40}$ » en lui faisant perdre «son caractère qualitatif, changeant et fluide» pour lui donner une forme spatialisée:

Il se fige en un continuum exactement délimité, quantitativement mesurable, rempli de "choses» quantitativement mesurables (les «travaux accomplis » par le travailleur, réifiés, mécaniquement objectivés, séparés avec précision de l'ensemble de la personnalité humaine): en un espace ${ }^{41}$.

À l'origine du pouvoir de réification du capital se trouve donc, chez Lukàcs, un procès de spatialisation du temps qui soumet ce dernier à la fixité et à l'absence de vitalité de l'espace. Suivant cette interprétation, Franck Fischbach a plus récemment soutenu qu'il fallait voir dans la subordination réelle du travail au capital, qui impose «la domination de la machine sur la force de travail», "l'expression visible de la domination de l'espace sur le temps ${ }^{42}$. Ainsi, la soumission de la temporalité de l'activité humaine concrète à l'espace constitué par le travail mort et le temps passé incorporés dans le capital fixe contraindraient «le travail, comme puissance temporalisante, créatrice de richesses et tournée vers l'avenir, [à] reproduire les conditions de sa propre domination par son autre, c'est-à-dire par l'espace ${ }^{43}$ ». La spatialité serait alors, dans le capitalisme développé, le facteur qui détermine la temporalité de la vie humaine en la niant.

S'il est vrai que la spatialité intervient dans la définition de la temporalité, peut-on pour autant en conclure qu'elle la domine? La relation de

40. G. Lukàcs, op cit., p. 117.

41. Ibid.

42. Franck Fischbach, «Comment le capital capture le temps», dans F. Fischbach (dir.), Marx. Relire Le Capital, Paris, PUF, 2009, p. 132.

43. F. Fischbach, La privation de monde..., op cit., p. 80. 
dépendance entre le temps et l'espace est-elle réellement unidirectionnelle? N'est-elle pas au contraire dialectique, comme le suggère plutôt la lecture qui vient d'être faite des écrits de Marx? Bien qu'il soit le support à la formation des plus-value absolue et relative, l'espace abstrait capitaliste ne crée pas lui-même ses propres conditions. Il est d'abord le produit de l'impératif temporel donné par la norme du temps de travail socialement nécessaire. Au stade de la soumission réelle, c'est du besoin de réduire cette norme temporelle que naît la nécessité de modifier le procès de travail afin de le rendre plus productif. Cette temporalité particulière participe donc elle aussi à la production de la spatialité qui lui permet de manifester son pouvoir de contrainte. La spatialisation du temps caractéristique du capitalisme contemporain s'accompagne alors à son tour d'une temporalisation de l'espace. Ces deux phénomènes concomitants prennent forme dans l'abstraction qu'ils produisent et qu'ils s'imposent l'un à l'autre. Le temps et l'espace abstraits apparaissent ainsi comme deux médiations centrales du capitalisme qui sont tout aussi essentielles à la reproduction de la domination par le capital qu'elles ne le sont l'une pour l'autre.

\section{L'actualité d'une théorie de l'abstraction spatio-temporelle pour comprendre l'actuelle financiarisation du capital}

Grâce à l'éclairage apporté par les travaux d'Henri Lefebvre sur l'espace, il est donc possible de faire une lecture des œuvres de maturité de Marx qui pose le caractère spatio-temporel de la dynamique sociale que l'on retrouve au cœur de la reproduction du capitalisme. Selon l'interprétation proposée dans ce texte, il semble que le développement du capital ne repose ni sur la domination du temps sur l'espace ni, à l'inverse, sur le primat de la spatialité sur la temporalité, mais plutôt sur le rapport d'interdépendance qui unit ces deux dimensions fondamentales de l'activité humaine dans et par lesquelles l'abstraction capitaliste se donne une consistance et une effectivité. La spatio-temporalité abstraite fournit les conditions de formation des médiations dans lesquelles le capital puise son pouvoir de contrainte : la valeur et le travail abstrait. Elle joue un rôle important dans le développement du capitalisme, et ce, d'une double façon. D'une part, la spatio-temporalité saisie par la dynamique abstraite-concrète du capital produit le cadre en perpétuelle transformation qui lui permet, comme le souligne Postone, de reconstituer en permanence les bases de sa propre expansion ${ }^{44}$. D'autre part, dans ce mouvement qui l'anime, elle réaffirme avec toujours plus de force les condi-

44. À la différence, cependant, que Postone a uniquement insisté sur la dimension temporelle de la dialectique de transformation-reconstitution sur laquelle il fonde son analyse du mouvement auto-expansionniste du capital. 
tions vitales du capital que sont le travail et la propriété privée et qui doivent demeurées inchangées pour en assurer la reproduction élargie ${ }^{45}$.

Le dialogue engagé dans ce texte entre Lefebvre et Marx jette ainsi les bases d'un cadre d'analyse qui, parce qu'il fait de l'abstraction spatio-temporelle une force motrice de la reproduction élargie du capitalisme, offre des possibilités de recherche intéressantes sur l'évolution actuelle du capitalisme. Le capitalisme contemporain (ou avancé) est fréquemment qualifié de "financiarisé » afin de souligner le rôle hégémonique et régulateur que joue l'accumulation financière dans sa reproduction. Le «nouveau capital financier» tire sa puissance de deux développements qu'il a connus depuis sa déréglementation progressive amorcée dans les années $1970^{46}$.

Premièrement, au cours de cette période, les relations financières se sont massifiées et largement diffusées au sein du salariat grâce à l'extension du crédit à la consommation et à l'apparition du fonds d'investissement comme «véhicule d'épargne ${ }^{47}$ ». Deuxièmement, œuvrant désormais dans un environnement libéralisé, les institutions financières ont développé puis mis en marché de nombreux produits permettant de transformer la masse de relations financières nouvellement créées en titres liquides, c'est-à-dire en titres échangeables en tout temps (en principe) ${ }^{48}$. Sous cette forme liquide, les créances accèdent à un univers de valorisation qui est tendanciellement (mais jamais entièrement) détaché (abstrait) de la spatio-temporalité inhérente au rapport entre créancier et débiteur qu'elles sous-tendent. C'est ainsi, par exemple, que les actionnaires d'une entreprise ou les créanciers d'un État peuvent, en tirant profit de la liquidité des titres qu'ils possèdent, spéculer sur leur valeur et formuler des exigences de rendements qui ont, en ellesmêmes, assez peu à voir avec la réalité de l'activité sur laquelle portent leurs créances.

L'exemple encore plus évocateur du pouvoir que la finance puise dans l'abstraction spatio-temporelle de la liquidité est sans doute celui des fameux produits financiers complexes à l'origine de la récente crise américaine des prêts subprime. Cette crise est survenue à la suite de l'effondrement d'un

45. En ce sens, je ne partage pas la conception de la propriété de Postone qui réduit celle-ci à une catégorie secondaire relevant exclusivement de la sphère de la distribution. En orientant uniquement son analyse vers la dimension distributive de la propriété, Postone néglige le rôle qu'elle joue dans la production en tant que mode exclusif d'appropriation de l'espace à l'origine du travail salarié.

46. Cette brève présentation des caractéristiques du phénomène de financiarisation du capitalisme est inspirée des travaux d'Éric Pineault. Voir entre autres Eric Pineault, Financiarisation, crédit et théorie critique du capitalisme avancé, dans F. Dupuis-Déri (dir.), Par-dessus le marché! Réflexions critiques sur le capitalisme, Montréal, Écosociété, 2012, 263 p.

47. E. Pineault, ibid., p. 68.

48. Au sujet de l'importance de la liquidité en tant qu'institution fondamentale de l'accumulation financière, voir André Orléan, Le pouvoir de la finance, Paris, Odile Jacob, 1999, 275 p. et André Orléan, L'empire de la valeur. Refonder l'économie, Paris, Seuil, coll. «La couleur des idées», 2011, 339 p. 
marché résidentiel dont la forte croissance des années précédentes avait en grande partie été soutenue par des opérations de titrisation hypothécaire. Ces opérations permettent à un émetteur de prêts hypothécaires de transformer ses créances en titres liquides afin de les vendre sur les marchés financiers. Elles transforment des capitaux immobilisés à long terme dans une résidence en occasion de valorisation rapide et transférable, c'est-à-dire en véhicule d'investissement immobilier beaucoup plus intéressant que sous sa forme d'origine. L'intérêt manifesté par les marchés financiers pour ces titres allégés des contraintes normalement associées aux créances hypothécaires (appelées en anglais mortgage backed securities) a par la suite incité les banques à assouplir leurs critères d'admissibilité, à prêter davantage et à créer par la même occasion les conditions favorables à la formation d'une bulle immobilière.

La crise qui s'en est suivie est donc largement attribuable à la possibilité que donne la titrisation d'abstraire la créance hypothécaire de la temporalité et de la spatialité particulières qui lui donnent naissance. L'acquéreur d'un titre ne se procure pas, à proprement parler, une hypothèque. Il achète un droit sur une portion de ses revenus éventuels. Ces droits peuvent circuler sans pour autant que la créance qui en est à l'origine (l'hypothèque) ne soit éteinte et libérée de son ancrage territorial. Chaque fois que les titres changent de mains, leur valeur financière est entièrement réalisée, contrairement à l'hypothèque d'origine qui ne génère des revenus qu'à long terme et progressivement (sur 25 et parfois même 40 ans). L'univers de valorisation dans lequel se déploie le capital financier investi dans le secteur de l'immobilier est ainsi fondé sur de nouvelles relations qui permettent de soustraire le temps et l'espace aux déterminations déjà abstraites de l'activité capitaliste, pour les soumettre à un nouveau procès d'abstraction (financiarisé). C'est dans ce second degré d'abstraction de l'activité sociale que prend forme la liquidité essentielle à la valorisation des titres et à la reproduction de la puissance du capital financier.

Bien qu'elles aient d'abord vu le jour sur le marché hypothécaire américain, les opérations de titrisation ne se limitent plus à ce seul secteur d'activité. Elles ont depuis été étendues aux dettes de cartes de crédit, aux prêts automobiles, aux marges de crédit garanties par l'avoir propre foncier ainsi qu'aux dettes d'études. Elles ont contribué à l'accroissement considérable de l'endettement des ménages et, par conséquent, à l'accélération de la tendance contemporaine à la massification des relations financières. La titrisation a même gagné le monde municipal où de nombreuses administrations (surtout américaines) ont désormais recours au financement fiscal par accrois- 
sement de la valeur foncière (Tax Increment Financing) ${ }^{49}$. Cette pratique permet aux villes de financer la revitalisation de leurs secteurs «déstructurés» par l'émission de titres garantis par la hausse anticipée des revenus fonciers que devrait générer leur remise à neuf. En d'autres termes, elle propose de fixer et de vendre aujourd'hui les retombées fiscales de l'embourgeoisement d'un quartier qui prendra des années à se réaliser, et ce, abstraction faite de tous les obstacles que les transformations spatiales projetées pourraient rencontrer.

Ces quelques exemples démontrent l'actualité de la problématique lefebvrienne de l'espace que l'on retrouve implicitement formulée chez Marx. Ils illustrent également toute la pertinence d'une étude de la financiarisation du capitalisme avancé qui se donnerait pour point d'ancrage la relation d'interdépendance entre le temps et l'espace ainsi que le procès d'abstraction auquel ils sont tous deux soumis.

49. La ville de Chicago, qui est un pôle international de l'innovation financière, fait d'ailleurs office de pionnière en la matière. Voir Rachel Weber, «Selling City Futures: The Financialization of Urban Redevelopment Policy» Economic Geography, vol. 86, no 3, 2010, p. 251-274. 